\title{
Microquasars: disk-jet coupling in stellar-mass black holes
}

\author{
I. Félix Mirabel $\dagger$ \\ European Southern Observatory, Alonso de Cordova 3107, Santiago, Chile \\ email: fmirabel@eso.org
}

\begin{abstract}
Microquasars provide new insights into: 1) the physics of relativistic jets from black holes, 2) the connection between accretion and ejection, and 3) the physical mechanisms in the formation of stellar-mass black holes. Furthermore, the studies of microquasars in our Galaxy can provide in the future new insights on: 1) a large fraction of the ultraluminous X-ray sources in nearby galaxies, 2) gamma-ray bursts (GRBs) of long duration in distant galaxies, and 3) the physics in the jets of blazars. If jets in GRBs, microquasars and Active Galactic Nuclei (AGN) are due to a unique universal magnetohydrodynamic mechanism, synergy of the research on these three different classes of cosmic objects will lead to further progress in black hole physics and astrophysics.
\end{abstract}

Keywords. Black hole physics - X-rays - binaries: jets - stars: general

\section{Introduction}

The physics in all systems that contain black holes is essentially the same, and it is governed by the same scaling laws. The main differences derive from the fact that the scales of length and time of the phenomena are proportional to the mass of the black hole. If the lengths, masses, accretion rates, and luminosities are expressed in units such as the gravitational radius $\left(R_{g}=G M / c^{2}\right)$, the solar mass, and the Eddington luminosity, then the same physical laws apply to stellar-mass and supermassive black holes (Sams et al. 1998; Rees 2003). For a black hole of mass $M$ the density and mean temperature in the accretion flow scale with $M^{-1}$ and $M^{-1 / 4}$, respectively. For a given critical accretion rate, the bolometric luminosity and the length of the relativistic jets are proportional to the mass of the black hole. The maximum magnetic field at a given radius in a radiation dominated accretion disk scales with $M^{-1 / 2}$, which implies that in the vicinity of stellar-mass black holes the magnetic fields may be $10^{4}$ times stronger than in the vicinity of supermassive black holes (Sams, Eckart \& Sunyaev 1998). In this context, it was proposed (Mirabel et al. 1992; Mirabel \& Rodríguez 1998) that supermassive black holes in quasars and stellar-mass black holes in X-ray binaries should exhibit analogous phenomena. Based on this physical analogy, the word "microquasar" (Mirabel et al. 1992) was chosen to designate compact X-ray binaries that are sources of relativistic jets (see Figure 1).

\section{Superluminal motions in microquasars and quasars}

A galactic superluminal ejection was observed for the first time in the black hole Xray binary GRS $1915+105$, at the time of a sudden drop at $20-100 \mathrm{keV}$. Since then, relativistic jets with comparable bulk Lorentz factors $\Gamma=1 /\left(1-\beta^{2}\right)^{1 / 2}$ as in quasars have been observed in several other X-ray binaries (Mirabel \& Rodríguez 1999; Fender

$\dagger$ On leave from CEA-Saclay, France. 


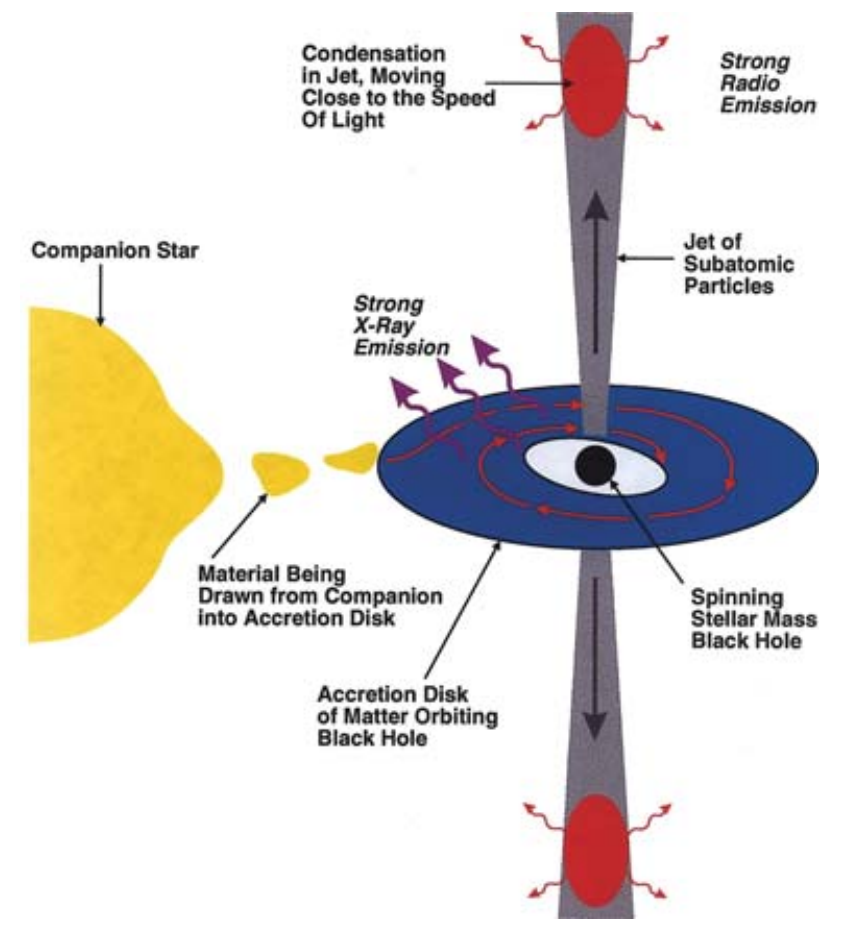

Figure 1. This diagram illustrates current ideas of what microquasars might be: compact objects - black holes and neutron stars - that are accreting mass from a donor star in $\mathrm{X}$-ray binary systems and ejecting plasma at relativistic speeds. The diagram not to scale.

2002; Paredes 2005). At present, it is believed that all X-ray accreting black hole binaries are jet sources.

Galactic microquasar jets usually move in the plane of the sky $\sim 10^{3}$ times faster than quasar jets and can be followed more easily than the latter (see Figure 2). Because of their proximity, in microquasars two-sided jets can be observed, which together with the distance provides the necessary data to solve the system of equations, gaining insight on the actual speed of the ejecta. On the other hand, in AGN located at $\leqslant 100 \mathrm{Mpc}$, the jets can be imaged with resolutions of a few times the gravitational radius of the supermassive black hole, as was done for M 87 (Biretta, Junor \& Livio 2002). This is presently not possible in microquasars, since such a precision in terms of the gravitational radius of a stellar-mass black hole would require resolutions of a few hundreds kilometers. Then, in terms of the gravitational radius in AGN we may learn better how the jets are collimated close to the central engine. In summary, some aspects of the relativistic jet phenomena associated to accreting black holes are better observed in AGN, whereas others can be better studied in microquasars. Therefore, to gain insight into the physics of relativistic jets in the universe, synergy between the knowledge of galactic and extragalactic black hole is needed.

\section{Accretion-jet connection in microquasars and quasars}

Microquasars have allowed to gain insight into the connection between accretion disk instabilities and the formation of jets. In $\sim 1$ hour of simultaneous multi-wavelength observations of GRS 1915+105 during the frequently observed 30-40 min X-ray oscillations in this source, the connection between sudden drops of the X-ray flux from the accretion disk and the onset of jets were observed on several occasions (Mirabel et al. 1998; Eikenberry et al. 1998); see Figure 3. 


\section{Quasar 3C279 \\ Microquasar GRS1915+105}
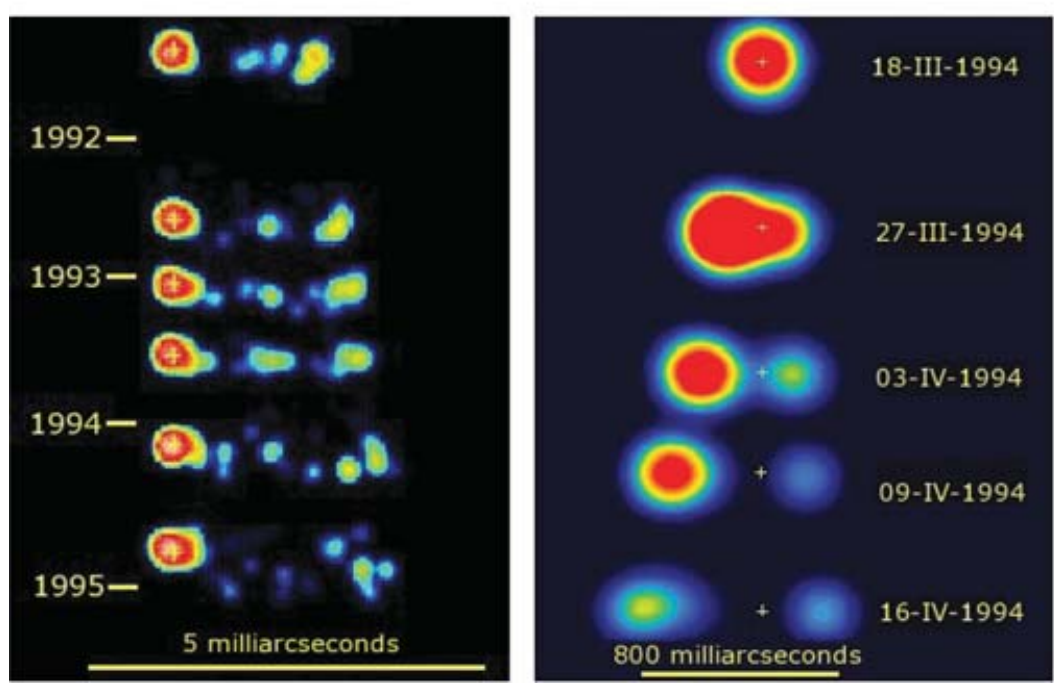

Figure 2. Apparent superluminal motions observed in the microquasar GRS $1915+105$ at $8.6 \mathrm{GHz}$ and in the quasar $3 \mathrm{C} 279$ at $22 \mathrm{GHz}$.

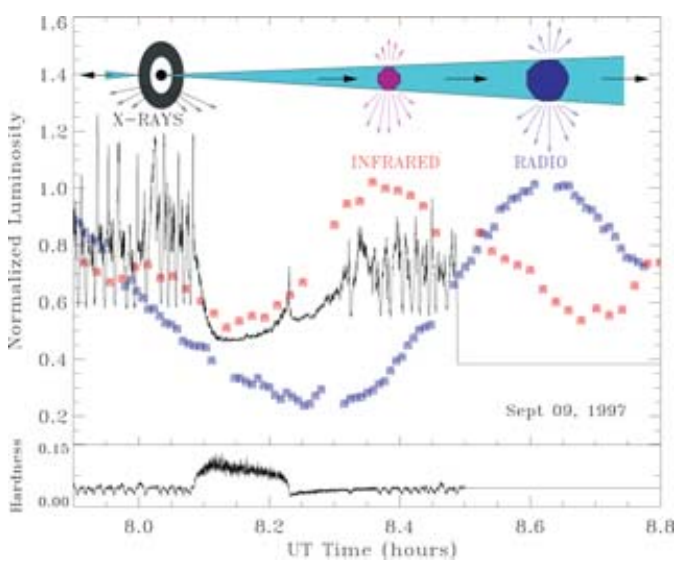

Figure 3. Direct evidence for the diskjet connection in the microquasar GRS 1915+105 (Mirabel et al. 1998). When the hot inner accretion disk disappeared, its X-ray brightness abruptly diminished. The ensuing X-ray recovery documented the inner disk's replenishment, while the rising infrared and radio emission showed plasma being ejected in a jet-forming episode. The sequence of events shows that material indeed was transfered from the disk to the jets. Similar transitions have been observed in the quasar 3C 120 (Marscher et al. 2002), but on time-scales of years, rather than minutes.

From these observations we have learned the following:

a) the jets appear after the drop of the X-ray flux;

b) the jets are produced during the replenishment of the inner accretion disk;

c) the jet injection is not instantaneous. It can last up to $\sim 10 \mathrm{~min}$;

d) the time delay between the jet flares at wavelengths of $2 \mu \mathrm{m}, 2 \mathrm{~cm}, 3.6 \mathrm{~cm}, 6 \mathrm{~cm}$, and $21 \mathrm{~cm}$ are consistent with the model of adiabatically expanding clouds that had been proposed to account for relativistic jets in AGN (van der Laan 1966).

e) synchrotron emission is observed up to infrared wavelengths and probably up to X-rays. This would imply the presence of electrons with $\mathrm{TeV}$ energies in jets;

f) VLBA images during this type of X-ray oscillations (Dhawan, Mirabel \& Rodríguez 2000) showed that the ejecta consist of compact collimated jets with lengths of $\sim 100 \mathrm{AU}$;

g) there is a time delay of $\sim 5$ min between the large drop of the X-ray flux from the accretion disk and the onset of the jets. These $\sim 5$ minutes of silence suggest that the compact object in GRS $1915+105$ has a space-time border, rather than a material border, namely, a horizon as expected in relativistic black holes. However, the absence of evidence of a material surface in these observations could have alternative explanations. 
After the observation of this accretion disk-jet connection in a microquasar, an analogous connection was observed in the quasar 3C 120 (Marscher et al. 2002), but on scales of years rather than minutes. This time-scale ratio is comparable to the mass ratio between the supermassive black hole in 3C 120 and the stellar black hole in GRS 1915+105, as expected in the context of the black hole analogy.

\section{References}

Biretta J. A., Junor W., Livio M. 2002, NewAR, 46, 239

Dhawan V., Mirabel I.F., Rodríguez L.F. 2000, ApJ, 543, 373

Eikenberry S. S., Matthews K., Morgan E. H., Remillard R. A., Nelson R. W. 1998, ApJ, 494, L61

Fender R. 2002, Lecture Notes in Physics, 589, 101

Marscher A. P., Jorstad S. G., Gómez J. L. et al. 2002, Nature, 417, 625

Mirabel I. F., Dhawan V., Chaty S. et al. 1998, A\&A, 330, L9

Mirabel I. F. \& Rodríguez L. F. 1998, Nature, 392, 673

Mirabel I. F. \& Rodríguez L. F. 1999, ARAA, 37, 409

Mirabel I. F., Rodríguez L. F., Cordier B. et al. 1992, Nature, 358, 215

Paredes J. M. 2005, in Radio Astronomy from Karl Jansky to Microjansky, eds. L. I. Gurvits, S. Frey \& S. Rawlings (EAS Publ. Ser.: Budapest), vol. 15, pp. 187-206 (astro-ph/0402671) van der Laan H. 1966, Nature, 211, 1131

Rees M. J. 2003, in Future of Theoretical Physics and Cosmology, ed. G. W. Gibbons et al. (Cambridge University Press: Cambridge), pp. 217-235 (astro-ph/0401365)

Sams B. J., Eckart A., Sunyaev R. 1998, Nature, 392, 673

Phil Charles: Could the apparent "dark jet" paradox of SS433 be answered by the high inclination which causes obscuration of most of the flux?

Felix Mirabel: Yes, the accretion disc produces high opacity to the X-rays and, as shown in the talk by Andrew King, the disc is probably highly warped. The source could be very bright if seen from a different direction.

VIRGINIA TRIMBLE: Suppose there have never been those jets in SS433, would there still be a supernova remnant there, or is the host nebula blown mainly by the activity of central source?

Felix Mirabel: This is an open question. Clearly, the lateral extentions seen in the nebula W 50 that hosts SS433, have been blown away by the jets.

Gregory Beskin: Is it possible to create jets without a black hole in the centre of the disc, for example by a neutron star?

Felix Mirabel: Yes indeed, there are jets in binaries with confirmed neutron stars. Clear cases are Scorpius X-1 and Circinus X-1.

Gloria Dubner: Comment on the previous question by V. Trimble: There is a way to probe if the whole bubble was created by a compact object. I believe that the whole nebula had been created by a supernova remnant and its shape was then distorted by the action of SS433's jets. The way to address this issue is by searching for spectral changes in the radio emission - the jets have a different spectrum than the rest of the bubble. 